\title{
Comparison of inhalational methoxyflurane (Penthrox®) and intramuscular tramadol for prehospital analgesia
}

\author{
Kegan Jianhong $\mathrm{Lim}^{1}$, MBBS, Zhi Xiong $\mathrm{Koh}^{2,3}$, BEng, Yih Yng $\mathrm{Ng}^{4,5}$, MBBS, MBA, Stephanie Fook-Chong ${ }^{3}$, MSc, \\ Andrew Fu Wah $\underline{\mathrm{Ho}}^{6}$, MBBs, Nausheen Edwin Doctor ${ }^{7}$, MBBS, Nur Ain Zafirah Mohd Said ${ }^{8}$, BSc, \\ Marcus Eng Hock Ong ${ }^{2,3}$, MBBS, MPH
}

INTRODUCTION Treatment of pain is an important component of prehospital care. Inhalational analgesia agents have attractive strengths, but there is a paucity of studies comparing these with more conventional agents. We aimed to compare inhalational methoxyflurane and intramuscular (IM) tramadol as first-contact analgesia in the Singapore national ambulance service.

METHODS Ambulances were randomised to carry either methoxyflurane or IM tramadol for the first six months and crossed over to the other arm after six months. Patients aged $\geq 16$ years, with acute pain arising from musculoskeletal trauma with Numerical Rating Scale (NRS) score $\geq 3$ were enrolled. Variables included NRS reduction, time variables, adverse effects, Ramsay Sedation Scores, and patient and paramedic satisfaction scores on a Likert scale.

RESULTS A total of 369 patients were enrolled into this study, but 26 patients were excluded due to missing data. The methoxyflurane arm had a shorter median time taken from arrival at the scene to drug administration ( 9.0 [interquartile range 6.0-14.0] minutes vs. 11.0 [interquartile range 8.0-15.0] minutes). For patients who achieved reduction in NRS $\geq 3$ within 20 minutes, those in the methoxyflurane arm took a shorter time. However, the methoxyflurane $(46.7 \%)$ arm experienced lower proportion of patients not achieving NRS reduction $\geq 3$ when compared to the tramadol ( $71.6 \%)$ arm after over 20 minutes. The methoxyflurane arm had significantly higher paramedic and patient satisfaction scores. CONCLUSION For the doses of medication used in this implementation study, methoxyflurane was superior in efficacy, speed of onset and administration, but had more minor adverse effects when compared to IM tramadol.

Keywords: analgesia, methoxyflurane, Penthrox, prehospital, tramadol

\section{INTRODUCTION}

Acute pain is common in prehospital medicine as a result of underlying trauma, burns or medical conditions. ${ }^{(1)}$ Nearly $20 \%-67 \%$ of patients transported by emergency medical services (EMS) to the hospital experience moderate or severe pain. ${ }^{(2,3)}$

There are several important reasons to treat pain promptly and adequately in the prehospital setting. Besides relieving suffering, analgesia reduces physiological and psychological stress, ${ }^{(4)}$ and facilitates extrication and therapeutic manoeuvres. ${ }^{(5)}$ In addition, prehospital pain management is associated with increased patient satisfaction. ${ }^{(6)}$ Indeed, the National Association of EMS Physicians issued a position paper advocating for the prioritisation of the relief of pain through developing prehospital protocols for assessment of pain and administration of analgesia. ${ }^{(7)}$ Nonetheless, there is mounting evidence that prehospital pain management may be inadequate. ${ }^{(3,8)}$

Each prehospital analgesic agent has unique practical advantages and disadvantages, and it is apparent that each EMS system must choose one that is most suited for its unique operation requirements and capabilities. Oral or intranasal medications are much easier to administer as compared to intramuscular (IM) or intravenous medications. The use of IM or intravenous administration also comes with the inherent risk of needle- stick injuries, inadvertent injection into a vein or extravasation of medication, if the techniques are poor. Opioid agents are classified as controlled substances in Singapore, and have a higher burden of accounting and auditing, security protocols and infrastructure to keep opioids under lock and key. There are also concerns over substance abuse among staff. For these reasons, opioid medications have not been used in Singapore EMS.

The Singapore EMS system had previously been using Entonox $₫$ (nitrous oxide and oxygen mixture) for prehospital analgesia, which presented problems, such as storage and maintenance issues due to the size of cylinders, poor mask fit, difficulty of some patients to activate the demand valve and encumbrance of carrying the Entonox cylinder into tight spaces. The national EMS medical advisory committee (MAC) had decided to phase out Entonox due to these issues, and identified IM tramadol and inhalational methoxyflurane as candidates to replace Entonox.

Methoxyflurane is a volatile anaesthetic agent of the fluorinated hydrocarbon group, with analgesic properties at subanaesthetic concentrations. It can be used as a selfadministered analgesic using a handheld inhalational device. While in widespread use in Australian EMS systems for the past three decades, ${ }^{\left({ }^{9}\right)}$ there is a paucity of good evidence to support its

\footnotetext{
${ }^{1}$ General Medicine, Tan Tock Seng Hospital, ${ }^{2}$ Department of Emergency Medicine, Singapore General Hospital, ${ }^{3}$ Health Services and Systems Research, Duke-NUS Medical School, ${ }^{4}$ Home Team, Ministry of Home Affairs, ${ }^{5}$ Emergency Medicine, Tan Tock Seng Hospital, ${ }^{6}$ Sing Health Emergency Medicine Residency, Singapore Health Services, ${ }^{7}$ Department of Emergency Medicine, Sengkang General Hospital, ${ }^{8}$ Medical Department, Singapore Civil Defence Force, Singapore

Correspondence: Prof Marcus Eng Hock Ong, Senior Consultant, Department of Emergency Medicine, Singapore General Hospital, Outram Road, Singapore 169608. marcus.ong.e.h@singhealth.com.sg
} 
use, in particular to compare its efficacy with non-placebo control patients. ${ }^{(10,11)}$ Tramadol is a weak $\mu$-opioid receptor agonist, and inhibits reuptake of serotonin and norepinephrine. ${ }^{(12)}$ It can be used by intravenous or IM routes, and has been used in the prehospital setting. ${ }^{(13,14)}$ Tramadol is classified as a non-narcotic analgesic in many jurisdictions. ${ }^{(14)}$ To our knowledge, there have been no published head-to-head studies that compared the efficacy of these two agents in any setting.

We hypothesised that in patients with acute pain (Numerical Rating Scale [NRS] pain score $\geq 3$ ) presenting to the ambulance service with musculoskeletal trauma (limb or back), pain relief by inhaled methoxyflurane would be equivalent to that by IM tramadol. We thus aimed to compare the analgesic efficacy of methoxyflurane and IM tramadol among patients presenting with acute pain in the prehospital setting, with respect to parameters, such as pain assessed by NRS at five minutes, ten minutes, 15 minutes and 20 minutes after start of the study treatment; time from arrival at scene to delivery of first analgesia; and time to onset of analgesia (arrival at scene to $\mathrm{a} \geq 3$-point reduction in pain scores, as assessed by NRS). Our secondary aims were to compare patients' satisfaction with regard to pain management, staff satisfaction and adverse effects with the use of the analgesic inhaler when compared to the use of IM tramadol for the treatment of acute pain.

\section{METHODS}

Paramedics in Singapore (equivalent to North American emergency medical technician-intermediate) are trained in basic life support, automated external defibrillator usage and adrenaline administration, and operate under offline medical oversight. ${ }^{(15)}$ In an earlier study, approximately $30 \%$ of patients with prehospital trauma in Singapore conveyed by paramedics in Singapore were classified under road traffic accident or other trauma. ${ }^{(16)}$ Tramadol is classified as a non-narcotic analgesic in Singapore. Before the commencement of this study, the existing local EMS practice for analgesia was to administer Entonox.

This trial was a prospective, phased, cluster-randomised crossover study. A simple randomisation procedure randomised fire stations into either the methoxyflurane or IM tramadol arm. Each fire station houses 1-3 ambulances. This procedure assigned 15 ambulances to carry methoxyflurane and another 15 to carry IM tramadol. After a six-month period, the ambulances from the two arms would cross over to carry the other study drug for another six months. The study period commenced in February 2014 and crossover occurred in August 2014. All patients were subsequently followed up by a review of their emergency department records for study drug-related adverse effects that occurred after the patient reached the hospital. Due to practical differences in the route of administration, there was no blinding of the participants to the allocated treatment. However, the data analyst was blinded to treatment allocation. This study was approved by SingHealth Centralised Institutional Review Board (CIRB), with waiver of informed consent (CIRB Ref: 2013/044/C). It was also registered via clinicaltrials.gov (identifier: NCT01887951).
All patients transported by Singapore EMS ambulances during the study period were recruited if they presented with acute pain arising from musculoskeletal trauma (upper and lower limbs, and back region) of NRS score $\geq 3$. Patients who were conscious, haemodynamically stable (systolic blood pressure/diastolic blood pressure $\geq 90 \mathrm{mmHg} / 60 \mathrm{mmHg}$ ), and aged $\geq 16$ years were also included.

The exclusion criteria for the trial were: pregnant or breastfeeding women and patients who had major head injury or impaired consciousness; acute intoxication or other conditions that may impair a patient's ability to score pain; chronic pain requiring long-term analgesia; and abdominal and chest pain.

Exclusion criteria for the methoxyflurane arm were: known renal or hepatic impairment; known or possible hypersensitivity to fluorinated anaesthetics in the patient or relatives; and concomitant use of nephrotoxic agents, such as gentamicin or tetracycline.

Exclusion criteria for the tramadol arm were: allergies to tramadol or codeine; history of seizures or epilepsy; taking antidepressants; on warfarin, heparin or novel anticoagulant agents (although antiplatelet agents were not an exclusion criterion); haemophilia; and other bleeding disorders, such as thrombocytopenia.

Before the start of the trial, all paramedics were trained to administer IM tramadol and to assist the patient to self-administer methoxyflurane. The training took place during sessions consisting of two lectures, video demonstration, hands-on practice and a multiple-choice quiz. A refresher online training was held before the crossover occurred.

In the methoxyflurane arm, patients were provided with one Penthrox $®$ inhaler (Medical Developments International, Springvale, Victoria, Australia). Penthrox is a whistle-shaped, hand-held inhaler, each primed with $3 \mathrm{~mL}$ of methoxyflurane. ${ }^{(17)}$ Each dose lasts approximately 25-30 minutes and a second dose can be added to extend the duration of action to 55-60 minutes. The recommended maximum total dose is $6 \mathrm{~mL}$ in 24 hours. A second inhaler of the same kind would be provided only upon request by the patient.

In the tramadol arm, patients were given IM injections of tramadol by the paramedics. Site of injection was the deltoid muscle. Each patient received a single dose of $1 \mathrm{~mL}$ of $50 \mathrm{mg} / \mathrm{mL}$ tramadol, which is the standard dose used in emergency departments in Singapore and was approved by the MAC.

Entonox was still kept on ambulances during the study period for backup analgesia, to be used when the ambulance had used up its supply of the study drug and could not wait for resupply, and for patients who did not meet inclusion and exclusion criteria for the study.

The primary outcomes were: (a) reduction in pain, as measured by reduction in NRS at five minutes, ten minutes, 15 minutes and 20 minutes after treatment; (b) time taken from arrival at scene to administration of treatment; and (c) time taken for onset of effective analgesia (to achieve a $\geq 3$-point reduction in NRS). The NRS is a self-reported pain assessment tool that has been validated in the acute ${ }^{(18)}$ and, specifically, prehospital settings. ${ }^{(19)}$ 


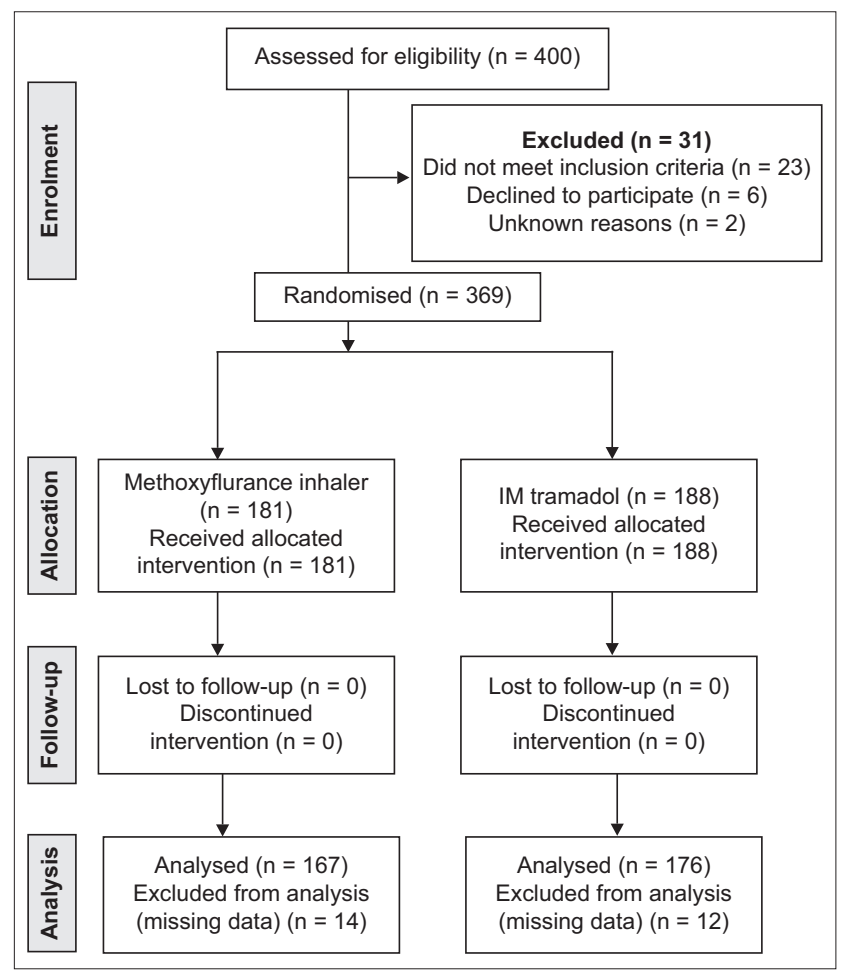

Fig. 1 CONSORT flow diagram shows patient enrolment into the study.

Secondary outcomes were: (a) Ramsay Sedation Score (RSS; Score 1: anxious and agitated or restless, or both; Score 2: cooperative, orientated and tranquil; Score 3: responds to commands only; Score 4: brisk response to painful stimuli or loud auditory stimulus; Score 5: sluggish response to painful stimuli or loud auditory stimulus; and Score 6: no response to painful stimuli or loud auditory stimulus) at five minutes, ten minutes, 15 minutes and 20 minutes; (b) paramedic satisfaction (based on ease of administration, speed of onset of analgesia and improvement of operating conditions) using a Likert scale; (c) patient satisfaction (based on effectiveness of relief and overall acceptance) using a Likert scale; and (d) adverse effects occurring in the prehospital setting and emergency department using a checklist.

Sample size calculation was based on an equivalence test of means for the primary outcome of drop in NRS from baseline at five minutes for a parallel-group design. To achieve a power of $80 \%$ at $5 \%$ significance level, when the true difference in NRS drop between the two arms was 0 , standard deviation of the drop in NRS was $1.5(\cong 1 / 6$ of range $0-10)$ and the required equivalence limits were -0.5 to +0.5 , a sample size of 155 for each arm was required.

Frequency tables and descriptive statistics for all outcome variables listed above were calculated. Associations between the treatment groups and all endpoints were analysed using $t$-test, chi-square test and Mann-Whitney $U$ test, and presented, where applicable. Statistical analyses were performed using IBM SPSS Statistics version 21.0 (IBM Corp, Armonk, NY, USA). A p-value $<0.05$ was considered to be statistically significant.

\section{RESULTS}

400 patients were screened in this study, but 23 patients did not meet the inclusion criteria and six patients declined to participate
Table I. Baseline characteristics of the study population.

\begin{tabular}{|c|c|c|}
\hline \multirow[t]{2}{*}{ Variable } & \multicolumn{2}{|c|}{ No. (\%) } \\
\hline & $\begin{array}{l}\text { Methoxyflurane } \\
(n=167)\end{array}$ & $\begin{array}{l}\text { Tramadol } \\
(n=176)\end{array}$ \\
\hline 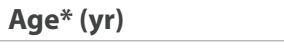 & $35(25-53 ; 16-91)$ & $39(27-53 ; 16-89)$ \\
\hline \multicolumn{3}{|l|}{ Gender } \\
\hline Male & $100(59.9)$ & $111(63.1)$ \\
\hline Female & $67(40.1)$ & 65 (36.9) \\
\hline \multicolumn{3}{|l|}{ Ethnicity } \\
\hline Chinese & $81(48.5)$ & $86(48.9)$ \\
\hline Malay & $39(23.4)$ & $43(24.4)$ \\
\hline Indian & $27(16.2)$ & $29(16.5)$ \\
\hline Other & $20(12.0)$ & $18(10.2)$ \\
\hline \multicolumn{3}{|l|}{ Site of injury } \\
\hline Abdomen & 0 & 1 \\
\hline Back & 39 & 37 \\
\hline Chest & 1 & 0 \\
\hline Face & 0 & 0 \\
\hline Head & 0 & 0 \\
\hline Upper limb & 61 & 64 \\
\hline Lower limb & 77 & 79 \\
\hline Neck & 0 & 0 \\
\hline Pelvis & 7 & 9 \\
\hline Other & 3 & 5 \\
\hline Multiple & 5 & 8 \\
\hline \multicolumn{3}{|l|}{ Past medical history } \\
\hline Not applicable & $82(49.1)$ & $106(60.2)$ \\
\hline Hypertension & $13(7.8)$ & 35 (19.9) \\
\hline Hyperlipidaemia & $13(7.8)$ & $17(9.7)$ \\
\hline Diabetes mellitus & $8(4.8)$ & $22(12.5)$ \\
\hline $\begin{array}{l}\text { Ischaemic heart } \\
\text { disease }\end{array}$ & $3(1.8)$ & $6(3.4)$ \\
\hline $\begin{array}{l}\text { Cerebral vascular } \\
\text { accident }\end{array}$ & $0(0)$ & $0(0)$ \\
\hline Asthma & $2(1.2)$ & $10(5.7)$ \\
\hline $\begin{array}{l}\text { Chronic obstructive } \\
\text { lung disease }\end{array}$ & $2(1.2)$ & $3(1.7)$ \\
\hline Renal impairment & $0(0)$ & $1(0.6)$ \\
\hline Liver disease & $0(0)$ & $0(0)$ \\
\hline Other & $22(13.2)$ & $23(13.1)$ \\
\hline Unknown & $3(1.8)$ & $3(1.7)$ \\
\hline $\begin{array}{l}\text { NRS on first } \\
\text { assessment* }\end{array}$ & $8.0(7.0-10.0 ; 4-10)$ & $8.0(7.0-9.0 ; 3-10)$ \\
\hline
\end{tabular}

*Data presented as median (interquartile range; range). NRS: Numerical Rating Scale

(two patients with unknown reasons). As shown in the CONSORT flow diagram (Fig. 1), a total of 369 patients were enrolled into this study, but 26 patients were excluded due to missing data. 167 patients in the methoxyflurane arm and 176 patients in the tramadol arm were finally analysed.

Table I shows the baseline characteristics of the patients enrolled. Patients in both arms were similar in terms of demographics and their site of injury, as well as median NRS on 
first assessment. The methoxyflurane arm had fewer patients with hypertension (methoxyflurane arm vs. tramadol arm: 7.8\% vs. $19.9 \%$ ), diabetes mellitus (methoxyflurane arm vs. tramadol arm: $4.8 \%$ vs. $12.5 \%$ )) and asthma (methoxyflurane arm vs. tramadol arm: $1.2 \%$ vs. $5.7 \%$ ) when compared to the tramadol arm.

The methoxyflurane arm experienced greater reductions in NRS at all four assessments post administration when compared to the tramadol arm (Table II), after five minutes (methoxyflurane arm vs. tramadol arm: 2.0 [interquartile range (IQR) 1.0-3.0] vs. 1.0 [IQR $0.0-2.0] ; p=0.001$ ), 10 minutes (methoxyflurane arm vs. tramadol arm: 3.0 [IQR 1.3-4.8] vs. 1.0 [IQR 0.0-2.0]; $p=0.001$ ), 15 minutes (methoxyflurane arm vs. tramadol arm: 3.0 [IQR 1.5-5.0] vs. 1.0 [IQR 0.0-2.0]; $p=0.001$ ) and 20 minutes (methoxyflurane arm vs. tramadol arm: 4.0 [IQR 1.5-5.0] vs. 1.0 [IQR 0.0-3.3]; $p=0.028$ ).

The methoxyflurane arm had a shorter median time taken from the paramedics arriving at the scene to the administration of the study drug when compared to the tramadol arm (Table II; methoxyflurane arm vs. tramadol arm: 9.0 [IQR 6.0-14.0] minutes vs. 11.0 [IQR 8.0-15.0] minutes; $\mathrm{p}<0.001$ ).

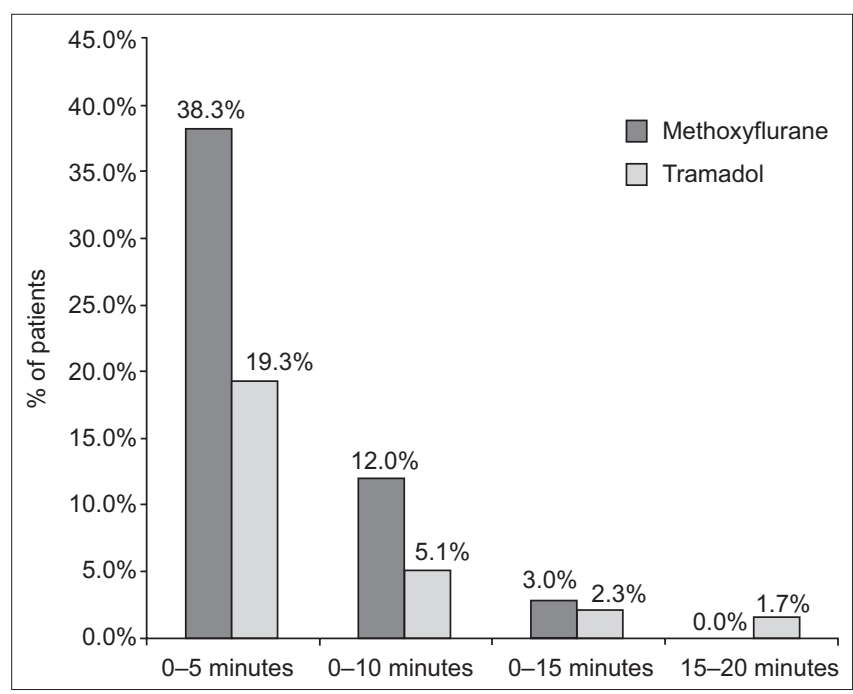

Fig. 2 Graph shows the time of onset of effective analgesia (reduction in NRS $\geq 3$ ) for the methoxyflurane and tramadol arms. NRS: Numerical Rating Scale
The methoxyflurane arm had a lower proportion of patients not achieving NRS reduction $\geq 3$ after more than 20 minutes when compared to the tramadol arm (Table II; methoxyflurane arm vs. tramadol arm: $46.7 \%$ vs. $71.6 \%$; $p<0.001$ ). Fig. 2 , which compares the time of onset of effective analgesia (reduction in NRS $\geq 3$ ) for the methoxyflurane and tramadol arms, shows that among patients who achieved NRS reduction $\geq 3$ within 20 minutes, those in the methoxyflurane arm took a significantly shorter time $(p<0.001)$. No patients required a second dose of methoxyflurane or backup analgesia in our study.

Table III shows the breakdown of adverse effects experienced following the administration of the study drugs. More patients in the methoxyflurane arm experienced one or more minor adverse effects when compared to the tramadol arm (methoxyflurane arm vs. tramadol arm: $44.3 \%$ vs. $6.3 \%$; $<0.001$ ). Drowsiness (methoxyflurane arm vs. tramadol arm: $31.7 \%$ vs. $2.8 \%$; $\mathrm{p}<0.001$ ) and headache (methoxyflurane arm vs. tramadol arm: $4.8 \%$ vs. $0.6 \%$; $p=0.014$ ) were experienced more commonly in the methoxyflurane arm.

On comparing patients for worse RSS, when assessed at five minutes, ten minutes, 15 minutes and 20 minutes, the methoxyflurane arm showed a trend towards having a higher (or worse) RSS when compared to the tramadol arm, although this was not statistically significant.

A breakdown of the paramedic and patient satisfaction survey scores on a Likert scale is presented in Table IV. In terms of paramedic satisfaction, methoxyflurane had significantly higher (or better) scores when compared to tramadol in all three areas: ease of administration ( $p=0.002)$; speed of onset $(p<0.001)$; and improvement of operating conditions $(p<0.001)$. In terms of patient satisfaction ( $p<0.001)$, methoxyflurane had significantly higher scores when compared to tramadol for effectiveness of relief, and a comparable score for patient acceptance.

\section{DISCUSSION}

In this real-world implementation study, inhalational methoxyflurane was superior to $50 \mathrm{mg}$ IM tramadol in terms of analgesic efficacy,

Table II. Time from arrival at scene to administration of study drug and proportion of patients achieving subsequent reduction of NRS $\geq 3$.

\begin{tabular}{|c|c|c|c|}
\hline \multirow[t]{2}{*}{ Variable } & \multicolumn{2}{|c|}{ No. (\%) } & \multirow[t]{2}{*}{ p-value } \\
\hline & Methoxyflurane $(n=167)$ & Tramadol $(n=176)$ & \\
\hline \multicolumn{4}{|l|}{ Reduction in NRS after administration* } \\
\hline After 5 min & $2.0(1.0-3.0 ; 0-8)$ & $1.0(0.0-2.0 ;-3-10)$ & $0.001^{\dagger}$ \\
\hline After $10 \mathrm{~min}$ & $3.0(1.3-4.8 ; 0-8)$ & $1.0(0.0-2.0 ;-2-10)$ & $0.001^{\dagger}$ \\
\hline After $15 \mathrm{~min}$ & $3.0(1.5-5.0 ; 0-8)$ & $1.0(0.0-2.0 ;-1-9)$ & $0.001^{\dagger}$ \\
\hline After $20 \mathrm{~min}$ & $4.0(1.5-5.0 ; 0-8)$ & $1.0(0.0-3.3 ;-1-9)$ & $0.028^{\dagger}$ \\
\hline Time taken to administer first dose of drug (min) & $9.0(6.0-14.0 ; 0-33)$ & $11.0(8.0-15.0 ; 2-44)$ & $<0.001^{\dagger}$ \\
\hline Patients who achieved NRS reduction $\geq 3$ at 5 -min intervals & & & $<0.001^{\dagger}$ \\
\hline $5 \mathrm{~min}$ & $64(38.3)$ & $34(19.3)$ & \\
\hline $10 \mathrm{~min}$ & $20(12.0)$ & $9(5.1)$ & \\
\hline $15 \mathrm{~min}$ & $5(3.0)$ & $4(2.3)$ & \\
\hline $20 \min$ & $0(0)$ & $3(1.7)$ & \\
\hline$>20 \min$ & $78(46.7)$ & $126(71.6)$ & \\
\hline
\end{tabular}

*Data presented as median (interquartile range; range). $\mathrm{tp}<0.05$ was statistically significant. NRS: Numerical Rating Scale 
Table III. Adverse effects after administration of methoxyflurane and intramuscular tramadol.

\begin{tabular}{|c|c|c|c|}
\hline \multirow[t]{2}{*}{ Adverse event } & \multicolumn{2}{|c|}{ No. (\%) } & \multirow[t]{2}{*}{ p-value } \\
\hline & Methoxyflurane $(n=167)$ & Tramadol $(n=176)$ & \\
\hline None & $106(63.5)$ & $158(89.8)$ & $<0.001^{*}$ \\
\hline Nausea and vomiting & $12(7.2)$ & $6(3.4)$ & 0.117 \\
\hline Retrograde amnesia & $0(0)$ & $0(0)$ & \\
\hline Drowsiness and dizziness & $53(31.7)$ & $5(2.8)$ & $<0.001^{*}$ \\
\hline Headache & $8(4.8)$ & $1(0.6)$ & $0.014^{*}$ \\
\hline Rash & $0(0)$ & $0(0)$ & \\
\hline Facial swelling & $0(0)$ & $0(0)$ & \\
\hline Cardiac arrest/respiratory depression & $0(0)$ & $0(0)$ & \\
\hline Throat irritation & $2(1.2)$ & $0(0)$ & 0.145 \\
\hline Cough & $1(0.6)$ & $0(0)$ & 0.304 \\
\hline Transient hypotension & $1(0.6)$ & $0(0)$ & 0.304 \\
\hline Other & $8(4.8)$ & $1(0.6)$ & $0.014^{*}$ \\
\hline Any adverse event & $74(44.3)$ & $11(6.3)$ & $<0.001^{*}$ \\
\hline Worse Ramsay Sedation Score ${ }^{\dagger}$ & & & 0.066 \\
\hline 1 & $9(5.4)$ & $9(5.1)$ & \\
\hline 2 & 109 (65.3) & $159(90.3)$ & \\
\hline 3 & $5(3.0)$ & $3(1.7)$ & \\
\hline 4 & $2(1.2)$ & $0(0)$ & \\
\hline 5 & $5(3.0)$ & $1(0.6)$ & \\
\hline 6 & $0(0)$ & $0(0)$ & \\
\hline
\end{tabular}

${ }^{*} p<0.05$ was statistically significant. $\mathrm{tn}=130$ for methoxyflurane arm (with 37 missing values) and $n=172$ for tramadol arm (with 4 missing values).

Table IV. Paramedic and patient satisfactions after administration of methoxyflurane and intramuscular tramadol using a Likert scale.*

\begin{tabular}{|llll|}
\hline Variable & \multicolumn{2}{c}{ Median (IQR; range) } & p-value \\
\cline { 2 - 3 } & Methoxyflurane $(\mathbf{n = 1 6 7 )}$ & Tramadol $(\mathbf{n}=\mathbf{1 7 6})$ \\
\hline Paramedic satisfaction & & & \\
\hline Ease of administration & $4.5(4.0-5.0 ; 2-5)$ & $4.0(4.0-5.0 ; 1-5)$ & \\
\hline Speed of onset & $4.0(3.0-5.0 ; 1-5)$ & $3.0(2.0-4.0 ; 1-5)$ & $<.002^{\dagger}$ \\
\hline Improvement of operating conditions & $4.0(4.0-5.0 ; 2-5)$ & $4.0(3.0-4.0 ; 2-5)$ & $<0.001^{+}$ \\
\hline Patient satisfaction & & & $<0.001^{+}$ \\
\hline Effectiveness of relief & $4.0(3.0-5.0 ; 1-5)$ & $3.0(3.0-4.0 ; 1-5)$ & $<0.001^{+}$ \\
\hline Patient acceptance & $4.0(3.0-5.0 ; 1-5)$ & $4.0(3.0-4.0 ; 1-5)$ & 0.258 \\
\hline
\end{tabular}

${ }^{*}$ On the Likert scale, $5=$ very satisfied and $1=$ very dissatisfied. $+\mathrm{p}<0.05$ was statistically significant. IQR: interquartile range

onset of effective analgesia and speed of administration. The strength of this study of methoxyflurane lies in its real-world trial design, which compared methoxyflurane with a viable alternative. The STOP! trial had compared methoxyflurane with a placebo and showed useful data to demonstrate its efficacy and safety. ${ }^{(10)}$

In the selection of a suitable analgesic agent for use in EMS, considerations other than a reduction in pain score are clearly important. The advantage of needleless administration within the small, confined cabin of a moving ambulance has clear safety advantages for both the patient and crew involved. The advantage of a quick onset of effective analgesia is magnified in an EMS system, with short prehospital times. In Singapore, the average transport time is less than 20 minutes and no patients in this study required a second dose of prehospital analgesia.

Our study also showed that there was a small risk of excessive sedation, with the use of both methoxyflurane and IM tramadol.
This emphasises the need for vigilance and pulse oximetry for monitoring patients who are administered these medications, in order to detect and manage possible respiratory depression.

Our study design lends itself to several limitations. First, the sample size, while calculated to detect a difference in the primary outcome, may not be powered to reliably detect rare adverse events. For example, there might have been a difference in the deterioration of RSS between the two arms that went undetected. Second, due to fundamental differences in the routes of administration of the two study drugs, neither the patient nor the paramedic could be blinded. Exclusion criteria for both arms were also slightly different. Third, the 50-mg dose of IM tramadol used might have been inadequate for some patients. The intention of using a slightly low dose was to ensure safety, as the Asian population tends to be of slighter build. $50 \mathrm{mg}$ IM tramadol is the standard dose used in 
emergency departments in Singapore for initial management of pain and this was the dosing approved by the MAC for this trial. This may be investigated in further studies, with graduated doses for different weight classes. Fourth, the population was relatively naïve to methoxyflurane and this study would not pick up adverse effects that were related to repeated exposure, which although rare, have been reported in the published literature with the use of full anaesthetic doses. ${ }^{(20)}$ Fifth, pain scores of patients after arrival at the emergency department were not available. Sixth, we did not collect some information, such as pulse oximetry and possible desaturation. This would have been useful, especially for patients who subsequently had sedation scores of 4 and 5. Nevertheless, as far as we are aware, no clinically important desaturations occurred in our patients. Finally, we took a pragmatic approach to real-world evaluation of the two treatments and this may not be a fair comparison of the drugs themselves. It was unsurprising that an IM injection had a longer onset than an inhaled agent.

Based on the results of this trial, MAC has recommended that methoxyflurane should be adopted as the first-line analgesic medication of choice for the ambulance service, with IM tramadol as the second-line medication for patients with contraindication to methoxyflurane. Entonox is being withdrawn from the local emergency ambulance service, as planned.

In conclusion, for the doses of medication used in this implementation study, inhalational methoxyflurane was superior to $50 \mathrm{mg}$ IM tramadol in terms of analgesic efficacy, onset of effective analgesia and speed of administration. It was also associated with better paramedic and patient satisfaction. However, it had a higher incidence of minor adverse effects, such as drowsiness and headache.

\section{ACKNOWLEDGEMENTS}

We would like to thank and acknowledge the contributions from all officers, paramedics and medics from the Medical Department, Singapore Civil Defence Force, who were involved in this study.

\section{REFERENCES}

1. Jennings PA, Cameron P, Bernard S. Epidemiology of prehospital pain: an opportunity for improvement. Emerg Med J 2011; 28:530-1.

2. McLean SA, Maio RF, Domeier RM. The epidemiology of pain in the prehospital setting. Prehosp Emerg Care 2002; 6:402-5.

3. Albrecht E, Taffe P, Yersin B, et al. Undertreatment of acute pain (oligoanalgesia) and medical practice variation in prehospital analgesia of adult trauma patients: a 10 yr retrospective study. Br J Anaesth 2013; 110:96-106.

4. Melzack R, Wall PD, Ty TC. Acute pain in an emergency clinic: latency of onset and descriptor patterns related to different injuries. Pain 1982; 14:33-43.

5. Mahoney PF, Carney CJ. Entrapment, extrication and immobilization. Eur J Emerg Med 1996; 3:244-6.

6. Studnek JR, Fernandez AR, Vandeventer S, Davis S, Garvey L. The association between patients' perception of their overall quality of care and their perception of pain management in the prehospital setting. Prehosp Emerg Care 2013; 17:386-91.

7. Alonso-Serra HM, Wesley K; National Association of EMS Physicians Standards and Clinical Practices Committee. Prehospital pain management. Prehosp Emerg Care 2003; 7:482-8.

8. Galinski M, Ruscev M, Gonzalez G, et al. Prevalence and management of acute pain in prehospital emergency medicine. Prehosp Emerg Care 2010; 14:334-9.

9. Buntine P, Thom O, Babl F, Bailey M, Bernard S. Prehospital analgesia in adults using inhaled methoxyflurane. Emerg Med Australas 2007; 19:509-14

10. Coffey F, Wright J, Hartshorn S, et al. STOP!: a randomised, double-blind, placebo-controlled study of the efficacy and safety of methoxyflurane for the treatment of acute pain. Emerg Med J 2014; 31:613-8.

11. Carley S, Body R. Methoxyflurane is a better painkiller than placebo: but do we want to know more? Emerg Med J 2014; 31:610.

12. Leppert W. Tramadol as an analgesic for mild to moderate cancer pain. Pharmacol Rep 2009; 61:978-92.

13. Ward ME, Radburn J, Morant S. Evaluation of intravenous tramadol for use in the prehospital situation by ambulance paramedics. Prehosp Disaster Med 1997; 12:158-62.

14. Vergnion M, Degesves S, Garcet L, Magotteaux V. Tramadol, an alternative to morphine for treating posttraumatic pain in the prehospital situation. Anesth Analg 2001; 92:1543-6.

15. Shin SD, Ong ME, Tanaka H, et al. Comparison of emergency medical services systems across Pan-Asian countries: a Web-based survey. Prehosp Emerg Care 2012; 16:477-96

16. Ho AF, Chew D, Wong TH, et al. Prehospital trauma care in Singapore. Prehosp Emerg Care 2015; 19:409-15.

17. Medical Developments International Limited. PENTHROX® (methoxyflurane) inhalation. Version 12. Available at: https://www.medicaldev.com/wp-content/ uploads/2015/07/Penthrox-Pl-v12.pdf. Accessed March 23, 2020.

18. Daoust R, Beaulieu P, Manzini C, Chauny JM, Lavigne G. Estimation of pain intensity in emergency medicine: a validation study. Pain 2008; 138:565-70.

19. Ismail AK, Abdul Ghafar MA, Shamsuddin NS, et al. The assessment of acute pain in pre-hospital care using verbal numerical rating and visual analogue scales. J Emerg Med 2015; 49:287-93.

20. Jephcott C, Grummet J, Nguyen N, Spruyt O. A review of the safety and efficacy of inhaled methoxyflurane as an analgesic for outpatient procedures. $\mathrm{Br}$ J Anaesth 2018; 120:1040-8. 